\title{
Método Avenzoar para la implantación racional de la atención farmacéutica en la farmacia comunitaria
}

\author{
Manuel Ojeda-Casares ${ }^{1,2,3}$, Purificación Casas Pérez ${ }^{1,2,3}$, María José de la Matta Martín ${ }^{1,2,3}$, \\ Enrique Ojeda-García ${ }^{1,2,4}$, Antonio M. Rabasco Álvarez ${ }^{1,3,5}$ \\ 1. Cátedra Avenzoar. 2. Farmacéutico comunitario en Sevilla. 3. Doctor en Farmacia 4. Máster universitario en atención farmacéutica y farmacia \\ asistencial. 5. Departamento de Farmacia y Tecnología Farmacéutica. Facultad de Farmacia. Universidad de Sevilla.
}

\section{PALABRAS CLAVE}

Metodología Avenzoar, metodología en atención farmacéutica, registro de pacientes, seguimiento farmacoterapéutico, farmacia comunitaria

\section{ABREVIATURAS}

AAF: atención

farmacéutica.

DM: dispensación de medicamentos.

FC: farmacia comunitaria. IF: indicación farmacéutica. PFT: perfil

farmacoterapéutico.

PG: programa de gestión.

PRM: problemas

relacionados con los medicamentos.

RE: receta electrónica.

RNM: resultados negativos a medicamentos.

SFT: seguimiento

farmacoterapéutico.

\section{KEYWORDS}

Avenzoar methodology, methodology in pharmaceutical care, patient registration, pharmacotherapeutic monitoring, community pharmacy

\section{RESUMEN}

Introducción: La evolución de la atención farmacéutica en España, el desarrollo de las tecnologías de la información y la comunicación, los proyectos de receta electrónica, la aparición de publicaciones como el Foro de Atención Farmacéutica, el desarrollo del Bot Plus y su integración con los programas de gestión de las farmacias comunitarias nos han permitido definir una metodología de trabajo en atención farmacéutica (AF), estratificada y selectiva.

Metodología: 1. Identificar el paciente destinatario del tratamiento. 2. Dispensación propiamente dicha. 3. Definición del perfil farmacoterapéutico de la farmacia. 3.1. Grupos terapéuticos. 3.2. Indicaciones terapéuticas. 4. Definición de patologías diana. 4.1. Relevancia numérica. 4.2. Relevancia terapéutica. 5. Definición del potencial de seguimiento farmacoterapéutico (SFT) de la farmacia comunitaria. 6. Elección de pacientes diana. 7. Ofrecimiento del servicio de SFT.

Resultados: En las cuatro farmacias comunitarias (FC) participantes se definió el perfil farmacoterapéutico a partir de los grupos terapéuticos a nivel 1. A partir de aquí dichas farmacias definieron sus respectivas patologías diana y los posibles pacientes a los que ofrecer el servicio. Se basan en dichos resultados para establecer la formación de sus profesionales.

Conclusiones: La metodología Avenzoar parte del conocimiento del paciente mediante el registro sistemático de las dispensaciones realizadas. Permite definir el perfil farmacoterapéutico de los pacientes. Permite decidir el nivel de implicación con el seguimiento de cada paciente según criterios previamente definidos. La metodología Avenzoar es compatible y complementaria con cualquier metodología de seguimiento farmacoterapéutico.

Avenzoar method for implementation of pharmaceutical care in community pharmacy

\section{ABSTRACT}

Introduction: The evolution of Pharmaceutical Care in Spain, the development of ICTs, e-prescribing projects, Bot Plus development and its integration with management programs of community pharmacies has allowed us to define a stratified and selective working methodology in Pharmaceutical Care.

Methodology: 1. Identification of the patient 2. Dispensation itself. 3. Definition of the pharmacotherapeutic profile of Pharmacy. 3.1. Therapeutic groups. 3.2. Therapeutic indications. 4. Defining target diseases. 4.1. Numerical relevance. 4.2. Therapeutic relevance. 5. Definition of potential pharmacotherapeutic monitoring of community pharmacy. 6. Selection of target patients. 7. Offering pharmacotherapeutic monitoring service.

Results and discussion: In the 4 participating pharmacies the pharmacotherapeutic profile was defined based on the treatment groups at level 1. From here, these pharmacies defined their respective target pathologies and potential patients who offer their services. On the basis of these results, pharmacies can establish their professional training.

Conclusion: The Avenzoar methodology is based on the systematic recording of dispensations made to patients. It sets the pharmacotherapeutic profile of patients. It allows you to decide the level of involvement with each patient as previously defined criteria. The Avenzoar methodology is compatible and complementary with any methodology of pharmacotherapeutic monitoring. 


\section{Introducción}

La atención farmacéutica (AF) en general, así como la dispensación de medicamentos (DM), la indicación farmacéutica (IF) o el seguimiento farmacoterapéutico (SFT), los conceptos de problemas relacionados con los medicamentos (PRM), resultados negativos a medicamentos (RNM), así como todos los consensos que se han alcanzado y publicado (1), son fruto de años de trabajo de muchos compañeros que en España llegaron a un punto de inflexión con los documentos de Foro de Atención Farmacéutica (2) y Foro de Atención Farmacéutica en Farmacia Comunitaria (3) publicados en 2008 y 2010, y que contaron con la participación de instituciones $\mathrm{y}$ asociaciones especialmente relevantes en esta materia. En estos documentos se recoge toda la experiencia acumulada en estos años. Pero lo más interesante es la apuesta de futuro que se realiza al reconocer que el futuro y desarrollo de la AF debe pasar porque los programas informáticos den solución a las necesidades de la misma, en su desarrollo, hasta el punto de definir las características que deben tener los programas informáticos que gestionen los distintos procesos de AF. Con ello se pretenden superar las barreras que tradicionalmente han dificultado el desarrollo de la $\operatorname{AF}(4,5)$ debido a la dificultad de explotación de los registros que se venían realizando de forma manual $(6,7)$, lo que focalizaba la AF en procesos individuales y aislados, y con serias dificultades en su explotación epidemiológica.

En paralelo, desde el año 2000, por los distintos profesionales implicados en el desarrollo del funcional del modelo andaluz de receta electrónica (RE), Receta XXI, se tenía claro que la RE era una herramienta que esencialmente no realizaba $\mathrm{AF}$, pero en su propio diseño se encerraba la posibilidad de potenciar de forma definitiva su implantación generalizada, permitiendo el registro de información sobre dispensaciones electrónicas y su asignación a los pacientes de forma prácticamente automática y muy ágil. De este modo, se permite la generación de un historial farmacoterapéutico del paciente, fruto del acto de dispensación, asumido como método generalizado de dispensación y por lo tanto elemento básico de esta metodología de implantación de la AF. Por todo ello, la integración de los programas de gestión (PG) utilizados por las farmacias comunitarias (FC) con la RE abría la posibilidad de realizar estos registros en el mismo acto de dispensación siempre y cuando dichos PG estuvieran preparados para procesar esta información de forma inmediata, para lo cual era imprescindible que, a su vez, estuvieran integrados o contuvieran en su interior programas de AF (8).

En este sentido, Bot Plus se ha ido constituyendo en el paradigma de programa de AF que reúne todas y cada una de las recomendaciones estipuladas en el documento de Foro de AF. Por todo ello, el paso siguiente fue poner en valor, por parte del Consejo General de Colegios Oficiales de Farmacéuticos, estas cualidades de Bot Plus y la conveniencia de que todos los PG que se utilizan en las FC españolas estuvieran integrados con esta herramienta informática, con el fin de que se beneficiaran de toda la base documental que abarca, tanto en medicamentos españoles o extranjeros, como en sus fichas técnicas, patologías, interacciones, alarmas, inclusión de protocolos de dispensación, registro de pacientes, intervenciones, etc. $(3,9)$.

Una vez conjuntadas todas estas herramientas se estaba en condiciones de proponer una metodología para facilitar la implantación de la $\mathrm{AF}$, racional y fundamentada en 3 aspectos: el perfil farmacoterapéutico (PFT) de la FC en cuestión, la estrategia a seguir por parte de la farmacia en AF y la definición del potencial de SFT de sus profesionales. El PFT anteriormente citado sería el responsable de describir las necesidades asistenciales de nuestro entorno poblacional.

Por tanto, el objetivo de este trabajo es proponer un sistema de implantación de la AF aplicable y compatible con todas las metodologías existentes.

Una de sus principales ventajas es que al poder realizarse de forma estratificada (10) permitirá a los profesionales de la FC decidir su grado de implicación en la misma, en función de su disponibilidad o de las características de la población a la que presten servicio. Además, por otra parte, se define un glosario de nuevos términos para posibilitar la apli- cación óptima de esta metodología. Mediante este trabajo se pretende así mismo establecer, en función de los datos recogidos sobre tratamientos de pacientes, patologías prevalentes, polimedicación y características demográficas, la estrategia a seguir por cada una de las farmacias participantes. De este modo, se definirán las patologías diana, la estrategia formativa e incluso el modo de seleccionar a los pacientes a los que ofrecer el servicio de SFC, e incluso SPD (11). Esta nueva sistemática de trabajo en $\mathrm{AF}$ se denominará "Metodología Avenzoar en Atención Farmacéutica”, dado que sus promotores pertenecen todos a la Cátedra Avenzoar de la Universidad de Sevilla, patrocinada por la Fundación Farmacéutica Avenzoar del Colegio de Farmacéuticos de dicha ciudad.

\section{Material y método}

Material

Software:

- Programa de gestión de FC UnycopWin.

- Bot Plus integrado en Unycop.

- Microsoft Office 2003 y siguientes.

- Receta Electrónica: Receta XXI versiones 2 y 3.

- Sistemas operativos Windows XP y 7.

- Internet Explorer, versiones $8 \mathrm{y}$ posteriores.

\section{Hardware:}

- Ordenadores personales.

- Portátiles.

- Impresoras.

\section{Método}

En el presente trabajo se ha contado con los datos provenientes de cuatro FC ubicadas en distintas zonas de Sevilla capital, lo que en principio podía presuponer resultados diferentes, hecho que se pretendía comprobar mediante el análisis objetivo de los datos que definieran el PFT de cada una de estas FC.

El estudio como tal es un estudio descriptivo que parte de la comparación de los distintos $\mathrm{PFT}$ de las FC participantes, y siendo dicho PFT un concepto evolutivo, no es necesario que la generación de los mismos sea coincidente en el tiempo. Y en relación con todo ello, comparar las distintas estrategias adoptadas por las 


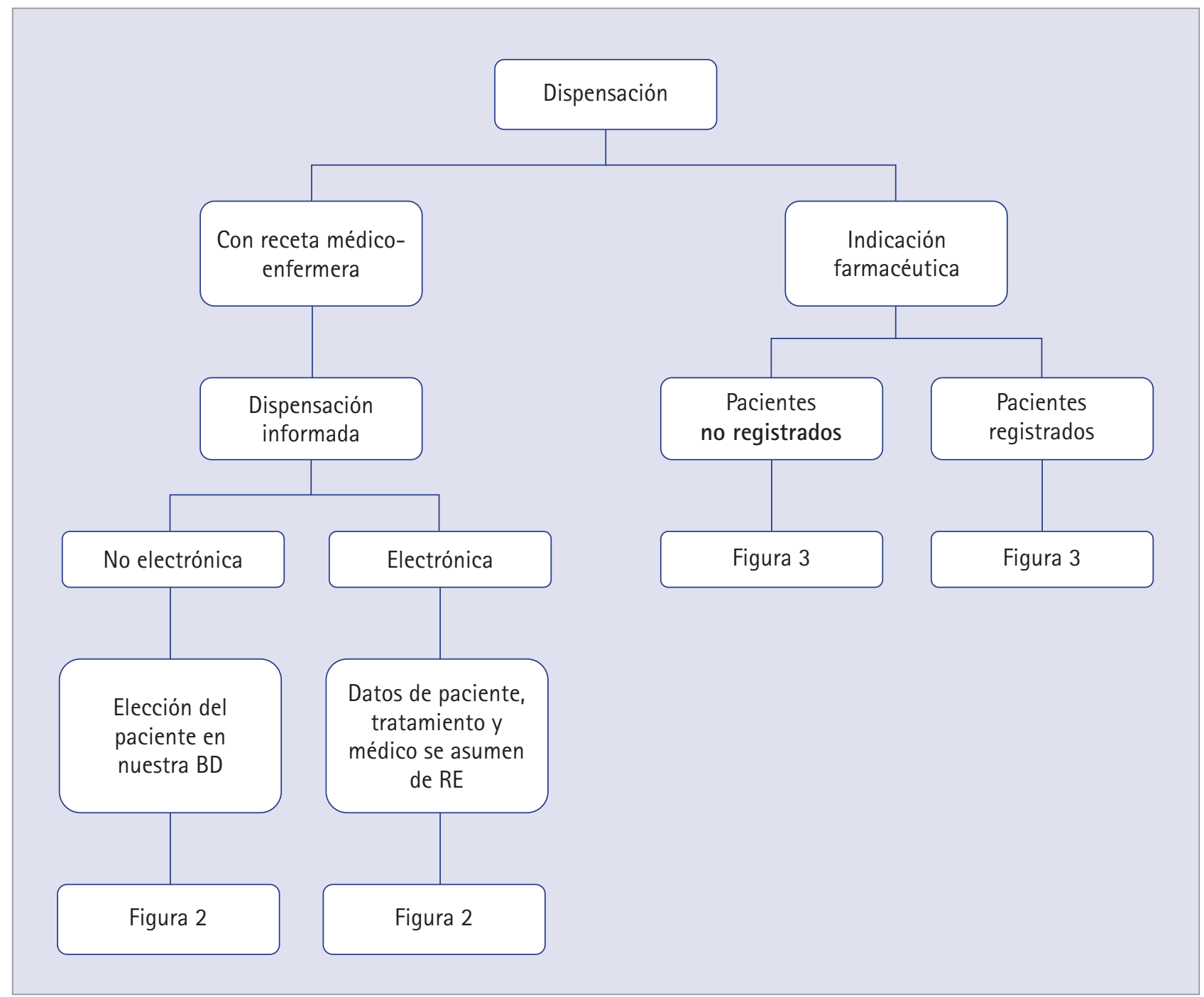

Figura 1 Estructuración del acto de dispensación

FC participantes, tanto en la aplicación de la AF como en la política formativa de sus profesionales o la estrategia en educación sanitaria a sus pacientes.

La metodología seguida en el presente trabajo es la que se ha denominado anteriormente como "Metodología Avenzoar en Atención Farmacéutica", la cual se describe a continuación. Tal como se definió en la Tesis Doctoral de Manuel Ojeda Casares (8), y que se rige por los principios que se recogen en el Foro de AF (3), consta de los siguientes items:

1. Identificación del paciente destinatario del tratamiento: se pueden dar dos supuestos:

1.1. El paciente está dado de alta en la base de pacientes: en este caso se busca en dicha base y se selecciona para asignarle su medicación. Cuando la dispensación se realiza mediante RE este proceso es automático.
1.2. El paciente no está dado de alta en la base de datos: en este supuesto lo primero que habrá que hacer es darlo de alta, después de que haya firmado el correspondiente consentimiento informado. Una vez registrados los datos mínimos para su identificación, continuamos tal como se hace en el supuesto anterior. El resto de la información se podrá completar posteriormente, mediante los datos aportados por el paciente en el consentimiento informado.

2. Dispensación propiamente dicha: tal como se recoge en la figura 1 , se pueden diferenciar dos grandes supuestos dependiendo de que la prescripción llegue con receta o bien se sustente en la indicación farmacéutica para sindromes menores.

2.1. Prescripción con receta: dispensación. En el primero de los casos, en el punto de venta es posible tener en una misma pantalla el tratamiento para varios pacientes diferentes, pertenecientes, por ejemplo, a una misma familia. Una vez aceptada la venta, antes de pasar al cobro de la misma, se despliega una nueva pantalla por cada uno de los pacientes identificados en el punto de venta. En ella aparece la medicación dispensable. A continuación se cumplimentan de forma ágil las preguntas necesarias para realizar este tipo de dispensación, siguiendo el árbol de decisiones recogido en la figura 2.

En ella se muestra el algoritmo de preguntas y decisiones que va realizando el farmacéutico durante la dispensación. Hay que señalar que dichos algoritmos están sustentados en unas pantallas dinámicas que varían según las respuestas registradas. En la figura se encuentran sombreadas en amarillo las respuestas que vienen predefinidas, ya que por su mayor frecuencia estadística se suelen dar más reiteradamente. Los registros básicos se realizan mediante 


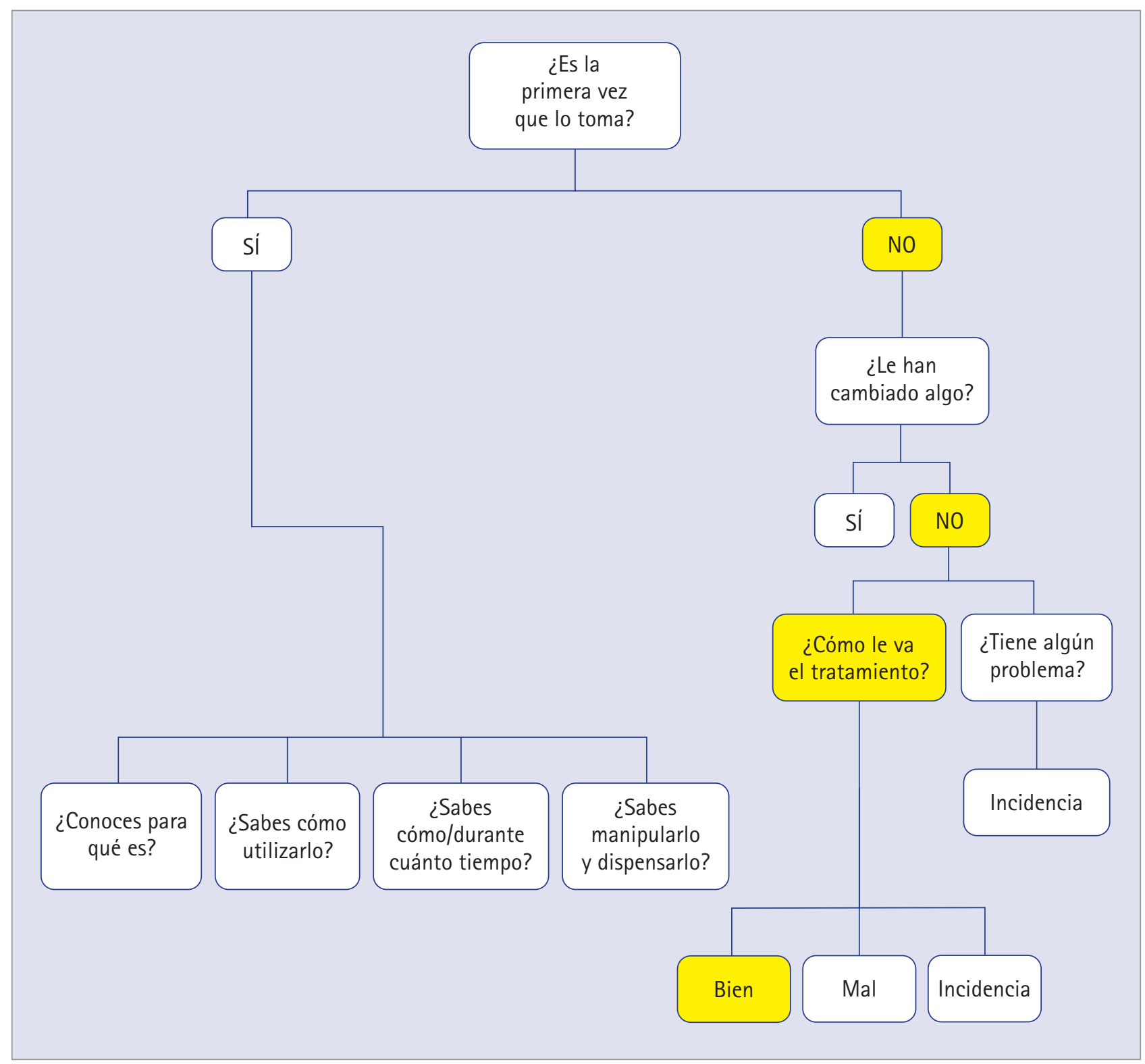

Figura 2 Algoritmos de actuación en la encuesta de dispensación. (En amarillo las respuestas predefinidas)

simples marcas, debiendo detenerse a reflejar incidencias y actuaciones, solamente aquellos casos que así lo requieran. Este procedimiento permite ir anotando las respuestas del paciente, de forma ágil, mientras se conversa con él, de forma distendida, durante la dispensación. De este modo, al cerrar la dispensación queda asociado el paciente a la medicación dispensada, en la fecha y hora que tuvo lugar, así como sus respuestas, actuaciones o incidencias registradas. $\mathrm{Y}$, además, quizás lo más interesante, datos todos ellos que pueden recuperarse y consultarse desde la ficha del paciente en cualquier momento posterior que se considere necesario.

En cualquier caso, a efectos de buena praxis y estadísticos, se pue- de realizar este algoritmo, de forma anónima, a todos los pacientes transeúntes o de paso, en el caso de que no interese tenerlos registrados como pacientes.

2.2. Dispensación mediante indicación farmacéutica (¿Qué me da para...?): en este modo de dispensación también se pueden encontrar los dos supuestos, pacientes anónimos o pacientes registrados.

2.2.1. En el caso de pacientes no registrados, si se decide seguir manteniéndolos anónimos se les debe preguntar sobre posible embarazo o lactancia, en caso de mujeres, así como por la existencia de otras patologías crónicas o agudas padecidas en el momento en que se está realizando la consulta. En caso afirmativo, se deberá especificar el tratamiento que siguen para dichas dolencias.

Seguidamente se les interpelará sobre la sintomatología para la que solicitan indicación. Una vez identificado el síndrome se aplicará el protocolo correspondiente para síndromes menores. $\mathrm{Si}$ éste indica que se puede realizar la dispensación, el sistema informático propondrá los posibles medicamentos dispensables para dicho síndrome menor.

2.2.2. En el supuesto de que el paciente se encuentre registrado en la base de datos, toda la información que le concierne es asumida por la herramienta informática en el primer bloque del cuestionario, referente a patologías y tratamientos del paciente ya recogidos en su ficha, especialmente su medicación habitual si la tuviera. 
A continuación, en el cuestionario sobre la sintomatología que se consulta se procede como ya se ha descrito en el apartado anterior.

Todo el procedimiento anteriormente descrito viene recogido gráficamente en la figura 3.

3. Definición del perfil farmacoterapéutico de la farmacia: el ir asignando la medicación al paciente en el acto de dispensación permite tener una información dinámica $\mathrm{y}$ actualizada de las necesidades de los pacientes según las patologías que padecen. Las herramientas informáticas permiten definir dicho perfil apoyado en:
3.1. Grupos terapéuticos:

- Edad.

- Sexo.

3.2. Indicaciones terapéuticas:

- Edad.

- Sexo.

4. Definición de patologías diana: a partir de la información obtenida en el apartado anterior, así como de las propias inquietudes de los profesionales de la farmacia comunitaria, se elegirán las patologías en las que se van a "especializar" dichos profesionales, lo que marcará la estrategia formativa y docente que se necesita. Esta elección se encuentra fundamentada en dos criterios básicos:
4.1. Relevancia numérica: se incide en aquellas patologías relacionadas con los grupos terapéuticos de mayor dispensación.

4.2. Relevancia terapéutica: se destacan aquellas patologías que, a pesar de no estar vinculadas a las dispensaciones más numerosas, son merecedoras de un seguimiento especial según criterio de los profesionales de la farmacia.

5. Definición del potencial de SFT de la farmacia comunitaria: los profesionales que en ella ejercen, en base a implicación y capacidad, definirán el número de pacientes a los que se les puede ofrecer el servicio de SFT.

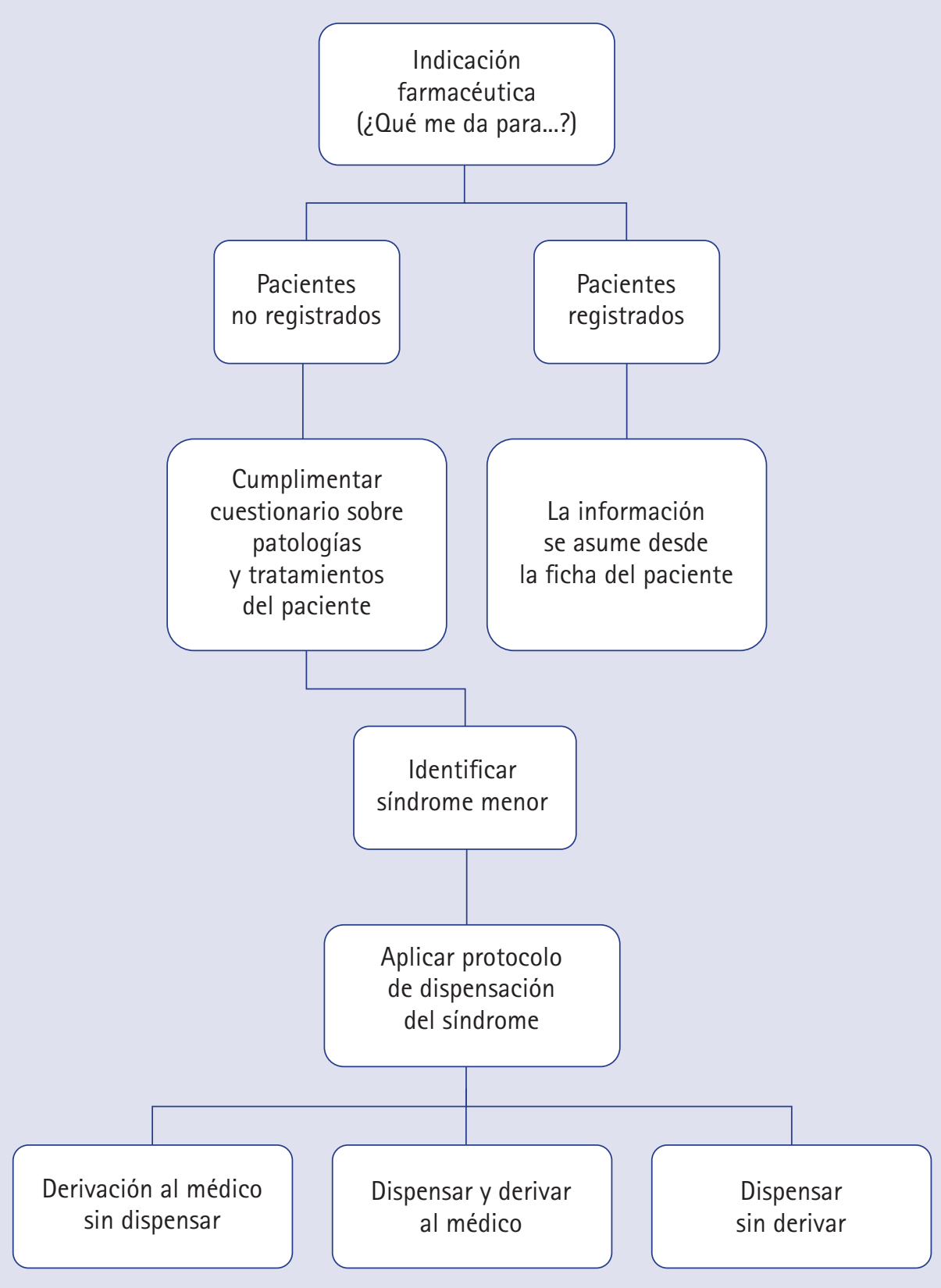

Figura 3 Procedimiento de dispensación mediante indicación farmacéutica 
6. Elección de pacientes diana: a continuación, se elegirán de entre los pacientes registrados, aquellos que por diversos criterios (fidelización, especial necesidad, idoneidad específica, etc.) se consideren objetivos preferentes para ofertarles el servicio de SFT.

7. Ofrecimiento del servicio de SFT: a los pacientes seleccionados se les citará en la farmacia comunitaria y se les ofrecerá el servicio de SFT una vez se les haya explicado los objetivos y el motivo por el que han sido seleccionados. En caso de conformidad por parte del paciente se procederá a explicarle el procedimiento que se va a seguir y se pacta con él un calendario de visitas.

\section{Resultados y discusión}

El presente trabajo como resultado de aplicar la "Metodología Avenzoar para Atención Farmacéutica" pone de manifiesto la particularidad de que los datos ya están recogidos y depositados en la base correspondiente, por lo tanto los resultados se presentaron conforme a la explotación que de los mismos se hizo según el trabajo o estudio que se decidió realizar. Lo que plantea la novedad de esta metodología frente a la que se viene desarrollando tradicionalmente mediante recogida de esa información con entrevistas a los pacientes (9).

Participaron en el desarrollo de esta metodología cuatro FC situadas en Sevilla capital. Dos de ellas, Torreblanca y San Jerónimo, han sido gestionadas por el mismo equipo de profesionales, pero en diferente ubicación, debido a que la FC de la que es titular Manuel Ojeda Casares, se trasladó desde el barrio de Torreblanca al de San Jerónimo. Las dos barriadas están situadas en la periferia del casco urbano de Sevilla, con la diferencia de que San Jerónimo está unida sin solución de continuidad a dicho casco urbano, mientras que Torreblanca está más separada del mismo, considerándose que pertenece a Sevilla capital por estar ubicada dentro de su término municipal. Sus habitantes son emigrantes venidos del mundo rural, y en su mayoría obreros más o menos cualificados como profesión, aunque muchos de sus descendientes han progresado en su formación y nivel cultural, habiéndose trasladado a otros barrios de la ciudad. En el caso de San Jerónimo, el perfil poblacional de la FC es totalmente distinto, pues se trata de una zona de nueva construcción, de viviendas de protección oficial destinadas a personas no mayores de 35 años. Constituye una zona joven de aluvión de otras zonas de la ciudad o provincia. Su nivel cultural es más elevado que el de Torreblanca o Padre Pío.

En el caso de la FC situada en el barrio del Porvenir cabe resaltar dos características esenciales. Este barrio está totalmente inmerso en el casco urbano de Sevilla, con un alto porcentaje de profesionales cualificados, pudiéndose enclavar dentro de un nivel de clase media alta, y un nivel cultural acorde a estas circunstancias. El segundo dato relevante a tener en cuenta en esta FC es que se encuentra próxima a un centro de salud, por lo que sus pacientes en un alto porcentaje son transeúntes. Por ello, la aplicación de la metodología Avenzoar nos aportará información muy útil sobre la capacidad de fidelización de la AF.

Y en el caso de la FC ubicada en la barriada Padre Pío, podemos repetir la descripción de las características poblacionales y demográficas de Torreblanca, al referirnos a su entorno poblacional. Está más cercana al casco urbano de Sevilla capital que la barriada de Torreblanca, pero está separada del mismo por una serie de vías de transporte de alto impacto, como son el entramado ferroviario de La Negrilla y la ronda de circunvalación SE-30. Esto conlleva una mayor dificultad de integración con el casco urbano de la ciudad a pesar de su relativa cercanía.

Como refuerzo gráfico de gran parte de lo antes expuesto, el módulo de AF del programa que se ha utilizado en las cuatro FC, UnycopWin, permite un análisis de la población del entorno basado en los datos de los pacientes registrados, distribuidos en rangos de edad y sexo. Se debe resaltar que estas gráficas no son estáticas sino que van evolucionando con el tiempo, ya que están basadas en los datos de las fichas de los pacientes, pudiendo producirse altas o bajas de los mismos por diversos motivos. Consideramos este hecho muy positivo ya que se encuentra permanentemente actualizado el entorno poblacional, con todas sus características, pudiendo detectar con facilidad todas sus necesidades.

Estas imágenes nos dan una primera información sobre los pacientes y sus requerimientos asistenciales, tanto desde el punto de vista de la medicación o educación sanitaria como de la demanda de servicios a prestar por su FC. Y, evidentemente, no tienen por qué ser los mismos los de una FC con respecto a otra. Pero ese análisis está más indicado en el estudio y caracterización de usuarios, que merece una publicación independiente, por sí solo.

Ya entrando de lleno en el análisis del perfil farmacoterapéutico de la FC, el primer indicador que se obtiene de nuestra base de datos de pacientes es el número de dispensaciones realizadas, distribuidas por grupos terapéuticos de nivel 1 (12). Los datos correspondientes a las FC objeto de nuestro estudio vienen recogidos en la tabla 1.

A continuación, una vez definido el perfil de nivel 1 , se seleccionan los grupos terapéuticos de mayor relevancia numérica y terapéutica. En cada uno de ellos se realiza el mismo análisis pero en este caso se analiza el número de dispensaciones en los subgrupos a nivel 3 de cada uno de ellos, procediendo una vez más a seleccionar aquellos que a criterio de los profesionales de la farmacia son más relevantes, combinando en este caso relevancia terapéutica y numérica. De la selección de estos subgrupos, mediante las herramientas que proporciona el programa de gestión, se genera un listado de pacientes, entre los que se eligen los pacientes diana con el objeto de ofrecerles el servicio de SFT por el profesional que se responsabiliza del mismo.

Aplicando todo este procedimiento en la farmacia de Torreblanca, se seleccionó por relevancia numérica los grupos C, N, A y M. Y por relevancia terapéutica el $\mathrm{B}, \mathrm{R}$ y L. Al hacer el análisis combinando relevancia numérica y terapéutica en los grupos de nivel 3, se concluyó seleccionando los subgrupos C09, N05, A10, B01, R03 y L04.

En el caso de la farmacia de San Jerónimo, por relevancia numérica, se seleccionaron los grupos $\mathrm{N}, \mathrm{A}, \mathrm{R}$ y C. Y por relevancia terapéutica, los grupos B, G, H y L. Al hacer el 
Tabla 1 Distribución de dispensaciones por farmacia y grupo terapéutico

\begin{tabular}{|c|c|c|c|c|}
\hline Farmacia Comunitaria & Torreblanca & San Jerónimo & Porvenir & Padre Pio \\
\hline \multicolumn{5}{|l|}{ Grupos terapéuticos } \\
\hline A: Tracto alimentario y metabolismo & 8.700 & 2.756 & 19.247 & 14.088 \\
\hline B: Sangre y órganos formadores de sangre & 2.996 & 728 & 6.282 & 3.995 \\
\hline C: Sistema cardiovascular & 10.575 & 1.306 & 24.254 & 17.081 \\
\hline D: Dermatológicos & 1.089 & 729 & 4.091 & 2.246 \\
\hline G: Sistema genitourinario y hormonas sexuales & 915 & 697 & 3.260 & 1.775 \\
\hline $\begin{array}{l}\text { H: Preparados hormonales sistémicos, excluyendo } \\
\text { hormonas sexuales e insulinas }\end{array}$ & 514 & 432 & 2.170 & 952 \\
\hline J: Antiinfecciosos para uso sistémico & 838 & 617 & 6.638 & 2.015 \\
\hline L: Agentes antineoplásicos e inmunomoduladores & 458 & 214 & 851 & 647 \\
\hline M: Sistema musculoesquelético & 3.203 & 1.218 & 9.544 & 5.608 \\
\hline N: Sistema nervioso & 9.208 & 3.301 & 26.350 & 19.686 \\
\hline P: Productos antiparasitarios, insecticidas y repelentes & 12 & 14 & - & - \\
\hline R: Sistema respiratorio & 2.829 & 1.791 & 12.344 & 6.213 \\
\hline S: Órganos de los sentidos & 1.499 & 475 & - & - \\
\hline V: Varios & 333 & 1.130 & - & - \\
\hline
\end{tabular}

análisis combinando relevancia numérica y terapéutica en los grupos de nivel 3, se escogieron los subgrupos N05, A10, R03, C09, B01, L01 y L04.

En la farmacia de El Porvenir se seleccionaron, por relevancia terapéutica, los grupos N, C, A, M y B. Y por relevancia terapéutica, R, L y J. Al hacer el análisis combinando relevancia numérica y terapéutica en los grupos de nivel 3, se concluyó seleccionando los subgrupos N05, C09, A10, M05, B01, R03, L01 y J07.
En la barriada Padre Pío se seleccionaron, por relevancia terapéutica los grupos N, C, A, M y B. Y por relevancia terapéutica R, L y J. Al hacer el análisis combinando relevancia numérica y terapéutica en los grupos de nivel 3, se concluyó seleccionando los subgrupos N05, C09, A10, M05, B01, R03, L04 y J07. En la tabla 2 se presenta la comparación de perfiles terapéuticos de las cuatro farmacias participantes.

En este punto cabe destacar que la metodología Avenzoar se basa en conceptos de relevancia numérica y relevancia terapéutica a partir del PFT basado en la distribución de las dispensaciones realizadas distribuidas por grupos terapéuticos, a diferencia de otros trabajos en los que se aborda el concepto de PFT (12).

A partir de este punto, las farmacias proceden a tomar decisiones sobre los planes de formación de cada uno de sus profesionales, orientada a las patologías seleccionadas y a los pacientes diana a los que se les ofrece el servicio.

Tabla 2 Comparación de perfiles terapéuticos de las 4 farmacias participantes

\begin{tabular}{|c|c|c|c|c|}
\hline Orden GT/Farmacia & Torreblanca & San Jerónimo & El Porvenir & Padre Pio \\
\hline $\mathbf{1}$ & C09 & N05 & N05 \\
\hline $\mathbf{2}$ & N05 & A10 & C09 & C09 \\
\hline $\mathbf{3}$ & A10 & R03 & A10 & M05 \\
\hline $\mathbf{4}$ & B01 & C09 & M05 & R03 \\
\hline $\mathbf{5}$ & R03 & B01 & B01 & R03 \\
\hline $\mathbf{6}$ & L04 & L01 & L01 & J04 \\
\hline $\mathbf{7}$ & & L04 & J07 \\
\hline $\mathbf{8}$ & & & L \\
\hline
\end{tabular}




\section{Conclusiones}

- La metodología Avenzoar se sustenta en la toma de decisiones basadas en el conocimiento del paciente mediante el registro sistemático de la dispensación informada que se le realiza cada vez que nos solicita medicación.

- Permite definir el perfil farmacoterapéutico de los pacientes que son atendidos en la farmacia.

- Permite decidir el nivel de implicación con el seguimiento de cada paciente, desde el registro de la dispensación informada hasta el seguimiento farmacoterapéutico de mayor o menor intensidad según criterios previamente definidos.

- La metodología Avenzoar es compatible y complementaria con cualquier metodología de seguimiento farmacoterapéutico.

\section{Agradecimientos}

Al Real e Ilustre Colegio de Farmacéuticos de Sevilla, a la Fundación Farmacéutica Avenzoar y a la empresa informática Unycop, por sus respectivas colaboraciones que han hecho posible la realización del presente trabajo.

\section{Referencias bibliográficas}

1. Foro de Atención Farmacéutica. Documento de Consenso. Madrid: Consejo General de Colegios Oficiales de Farmacéuticos; 2008.

2. Foro de Atención Farmacéutica en Farmacia Comunitaria. Farmacéuticos Comunitarios 2010;2(2):80-82.

3. Ojeda-Casares M. El impacto de las nuevas tecnologías en la consolidación del modelo farmacéutico español: desde la Red Corporativa Farmacéutica hasta la expansión de la Atención Farmacéutica, pasando por la Receta Electrónica [Tesis doctoral]. Sevilla: Universidad de Sevilla; 2010.

4. Casas P, Martín MJ. Registro en dispensación. Integración de un protocolo informático de dispensación en el programa de gestión de la farmacia comunitaria. Farmacéuticos Comunitarios 2011;3(2):52-57.

5. Agencia Española del Medicamento. Listados de principios activos por grupos ATC e incorporación del pictograma de la conducción. [Internet]. Madrid. Actualizada 12/03/2014. [consultada 03/04/2014]. Disponible en: http://www. aemps.gob.es/industria/etiquetado/conduccion/listadosPrincipios/home.htm.

6. Martín-Calero MJ, Machuca M, Murillo MD, Cansino J, Gastelurrutia MA, Faus MJ. Structural process and implementation programs of pharmaceutical care in different countries. Curr Phar Design. 2004;10: 3969-3985. doi:10.2174/1381612043382549
7. Moranta F, Maroto A, Sitjar T, Sanz M. Registro rápido de actuaciones farmacéuticas en farmacia comunitaria. e-Farmacéutico Comunitario 2006; Extra 2006: 30.

8. Rodríguez MJ, Pérez Accino C, Allú V, Domingo T, Fernández Montes T, Gamarra C, et al. Registro de las intervenciones del farmacéutico en la dispensación activa: resultados. Seguimiento Farmacoterapéutico 2004;2(1):29-31.

9. Hidalgo J, Cámara D, Baena MI, Fajardo PC, Martínez-Martínez F. Barreras para la implantación del seguimiento farmacoterapéutico en las farmacias comunitarias de Granada (España). Seguimiento Farmacoterapéutico 2005;3:144-149.

10. Gastelurrutia MA, Fernández-Llimos F, Benrimoj SI, Castrillón CC, Faus MJ. Barreras para la implantación de servicios cognitivos en la farmacia comunitaria española. Aten Primaria. 2007;39:465470. doi:10.1157/13109494

11. Silva MM, Tuneu L, Calleja MA, Faus MJ. Situación del seguimiento farmacoterapéutico en la atención hospitalaria. Pharm Care Esp. 2008;10(4):171-192.

12. Gastelurrutia MA, Feletto E, Noain MA, Martínez-Martínez F, Varas R, Benrimoj S. Exploración de las percepciones de los farmacéuticos comunitarios en la práctica del seguimiento farmacoterapéutico dentro del contexto del programa conSIGUE. Pharm Care Esp. 2011;13(6):280-288. 